\title{
Full English Immersion Teaching of Electromagnetism of University Physics \\ Huaqiu Deng
}

\author{
School of Physics and Optoelectronics, South China University of Technology, Guangzhou 510640, \\ China \\ eehqdeng@scut.edu.cn
}

Keywords: Electromagnetism, University physics, Full English immersion teaching

\begin{abstract}
This paper applies full English immersion teaching on electromagnetism of university physics, discusses graphic method, comparison method and discussion method of full English immersion teaching, and shows that the full English immersion teaching is an effective one in teaching of electromagnetism of university physics.
\end{abstract}

\section{Introduction}

University physics is a public basic course in universities of science and technology, involved into mechanics, thermodynamics, electromagnetism, optics and atomic physics, etc, and lays the foundation for students to study further in the future. At present the world get closer and closer. In order to adapt to the new situation of China's accession to the WTO, and to meet the international challenges, the Ministry of Education of China pointed out that "Bilingual teaching is our important way for higher education to adapt to the trend of economic globalization, to develop export-oriented talents with the consciousness of the international cooperation and ability of international communication and competition."[1], various universities gradually carried out bilingual teaching[2] and the full English teaching to meet the strong demand for high-quality comprehensive talents. This paper carried out the study of full English immersion teaching on the electromagnetism of university physics.

\section{Immersion Teaching}

The government of Canada supported the French immersion program in northern area to promote the fusion and cultural exchange between English and French during the 1960s. Immersion teaching is a teaching method that students are placed in a second language environment, major courses are taught in second language, and students naturally gain the ability of second language from the professional courses taught in second language. It has the following characteristics[3-5]

(1) The second language is the medium of teaching.

(2) Immersion courses and native language courses won't conflict.

(3) Immersion teaching supports native language.

(4) Immersion teaching has the function of bilingual teaching.

(5) Immersion teaching is usually confined to the classroom.

(6) Students should have the same or similar level in second language.

(7) Teacher should have the ability of bilingual teaching.

(8) Class culture is still of the native culture

Because immersion teaching has training roles for gaining foreign language ability and professional ability, it was introduced to North America and wider region during 1970s and 1980s, and was also studied in China[6-8]. 


\section{Full English Immersion Teaching of Electromagnetism}

University physics involves mechanics, thermodynamics, electromagnetism, optics and atomic physics, etc[9-12]. Objects are different, systems are also different, and professional English vocabulary is multifarious. The requirements for full English teaching are that coursewares are written in English, teachers teach in English, students ask questions in English, teachers answer questions in English, students do their homework in English and take the final exam in English. That is why English are widely used in full English teaching for university physics teaching. Because students' native languages are Chinese, not English, the students' logical thinking was conducted in Chinese, the effect of the full English teaching depends on whether teachers can give clear illustration of university physics knowledge in English, whether students can understand the knowledge. How to make full English teaching of university physics with high quality? Immersion England teaching is an effective way. Let the student immersed in the English environment who forget Chinese temporarily and think in English ASAP, students can follow up the full England teaching, ask, answer and discuss in English, even though they are not familiar with English vocabulary of various parts of university physics.

Electromagnetism are two important parts of mechanics of university physics, the goal to teach the two parts is to make the students understand theory and application of electromagnetism. Translation is usually taught first, then rotation. To enhance the effect of immersion English teaching is through illustration, comparison and discussion.

Full English immersion teaching of electromagnetism by graphics is to use tools such as multimedia, to enhance the features of graphics and text, to clearly illustrate the concepts and essence of electromagnetism in English, to help students to create direct sensation and to understand relevant English vocabulary of electromagnetism.

There is interaction between two distant static charges. In order to understand the interaction, a field is usually created around a charge which depends on the charge, position and the medium around it. When there is another charge existing inside the field, the field will apply a force on the charge. The field is static electric field which is a vector with magnitude and direction. For easy understanding, an electric line is used to represent the electric field, and its tangent direction on the electric line will express the direction of electric field at that point. A positive charge will create an electric field pointing radius as shown in Fig.1(a), and a negative charge will create an electric field pointing towards it as shown in Fig.1(b) .

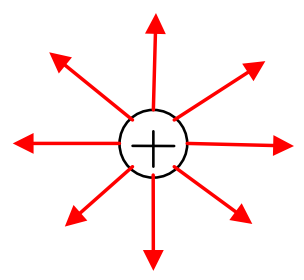

(a)

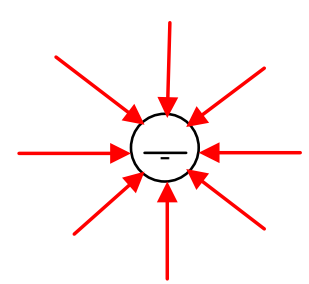

(b)

Fig.1 Electric field created by (a) positive charge and (b) negative charge

A moving charge particle will create a field around it as shown in Fig.2. A positive charged particle will create a magnetic field which follows right-hand role: a thumb points to the direction of movement, other four fingers will point to the direction of the magnetic field. A positive charged particle will create a magnetic field which follows right-hand role: a thumb points to the direction of movement, other four fingers will point to the direction of the magnetic field. A positive charged particle will create a magnetic field which follows right-hand role: a thumb points to the direction of movement, other four 
fingers will point to the direction of the magnetic field. The field will apply a force on a moving charged particle which is perpendicular to the plane created by the moving direction of the charged particle and the pointing from the charged particle to another moving charged particle. The medium for the interaction between two moving charged particles is a magnetic field. A magnetic field is a vector with magnitude and direction. A moving positive charged particle will create a magnetic field which follows right-hand role: a thumb points to the direction of movement, other four fingers will point to the direction of the magnetic field. A moving negative charged particle will create a magnetic field which follows anti-right-hand role: a thumb points to the reversed direction of the movement, other four fingers will point to the direction of the magnetic field.

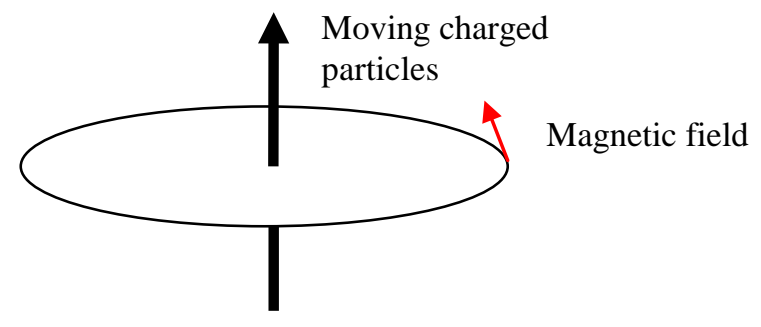

Fig.2 Magnetic field

Full English immersion teaching of electromagnetism by comparison is to compare the same and differences between electronic field and magnetic field, to let students understand the principles, be familiar with professional English vocabulary, and grasp knowledge key points of these two movements.

Electric field is usually taught first, then magnetic field in full English teaching of university physics. How to let students grasp knowledge points of magnetic field during full English teaching of it? Though electric field and magnetic field are two different fields, students are immersed in full English teaching of electromagnetism, compare the same and difference of electromagnetism, understand their principles, get familiar with relevant professional English vocabulary, and grasp their knowledge key points.

Table 1 is the comparison between electric field and magnetic field. It can be seen from the Table 1 that physical quantities are one-to-one correspondence, and students can quickly grasp the key knowledge points of electromagnetism.

Table 1. Comparison between electric field and magnetic field

\begin{tabular}{|c|c|}
\hline Electric field & Magnetic field \\
\hline Static charge $q$ & Velocity $\vec{v}$ \\
\hline Electric field $\vec{E}$ & Magnetic field $\vec{B}$ \\
\hline Electrostatic Force $\vec{F}_{E}=q \vec{E}$ & Magnetic force $\vec{F}_{B}=q \vec{v} \times \vec{B}$ \\
\hline
\end{tabular}

Full English immersion teaching of electromagnetism by discussion is to preset discussion topics of electromagnetism, let students be immersed in English and discuss these topics in order to promote the effect of full English teaching, and know how the students understand about electromagnetism.

Because university physics involves mechanics, thermodynamics, electromagnetism, optics and atomic physics, and is usually finished within two semesters with 96 or 128 teaching hours, it is not suitable to have long hour discussion.

Discussion topic of electromagnetism depends on discussion hour. Simple discussion topic can be set for 15 minutes long discussion, such as "Please discuss the characteristics of electrostatic force and magnetic force”, etc. More difficult discussion topics can be set for 30 minutes discussion, such as 
"Please discuss the creation of electric field and magnetic field", etc. Students will gain oral communication ability and understand deeply about electromagnetism through discussion. Teachers can participate into the discussion to know how students understand electromagnetism and give more clear illustration about electromagnetism.

\section{Summary}

Electromagnetism is an important topic of university physics. Teaching effect can be promoted through full English immersion teaching by graphics, comparison and discussion, which is affirmed by students of Grade 2012 and Grade 2013 of Full English Classes of Civil Engineering and Environmental Engineering, South China University of Technology.

\section{Acknowledgement}

This work is partially supported by "Research and Practice on Constructing Full English Teaching Model Course - University Physics” of Guangdong Education Bureau and by "Research on Full English Immersion Teaching of University Physics” of South China University of Technology.

\section{References}

[1] Chinese Ministry of Education: Article 4 (2001)

[2] DENG Hua-qiu: Theory and Practice of Education, Vol. Monography (2007)

[3] Evans, T.D., Maggie Jo St John. Development in ESP: A Multi-disciplinary Approach (Cambridge University Press, 1998)

[4] Merrill Swan: Annual Review of Applied Linguistics, Vol.20 (2000), p.1999-222.

[5] Walker, C.L., Diane J. Tedick: The Modern Language Journal, No.1(2001)

[6] DU Feng-feng: International Economics and Trade Research, Vol. Monography (2005)

[7] LU Ming-zhi: International Economics and Trade Research, Vol. Monography (2005)

[8] CAO Chang-mei, GUO Guo-qing, SHEN Wei-zai: Chinese Journal of Clinical Anatomy, Vol.26 (2008)

[9] ZHANG Li-bin, ZHANG Gong: College Physics, Vol.30 (2011), p. 48-64

[10] Xiaolin SHU, Shuo JIN, Ying ZHANG, Hongbo ZHOU, Guanghong LV, in: 2012 Conference on Creative Education (2012)

[11] Shi Jian-hua Liang Hong, in: 2010 Third International Conference on Education Technology and Training (ETT) (2010)

[12] Halliday \& Resnick, Jearl Walker: Fundamentals of Physics (9th Edition, John Wiley \& Sons, Inc ) 